\section{MOTH DIVERSITY OF TAWANG DISTRICT, ARUNACHAL PRADESH, INDIA}

\author{
Kailash Chandra ${ }^{1} \&$ S. Sambath ${ }^{2}$
}

${ }^{1}$ 1. Zoological Survey of India, Prani Vigyan Bhavan, 535, 'M' Block, New Alipore, Kolkata, West Bengal 700053, India

${ }^{2}$ Zoological Survey of India, Central Zone Regional Centre, 168-169, Scheme No. 5, Vijay Nagar, Jabalpur, Madhya Pradesh 482002, India

${ }^{1}$ kailash611@rediffmail.com (corresponding author)

2sambath63@gmail.com

Recent estimates report over 1,27,000 species of moths from all over the world (Alfred et al. 1998). Of which, over 12,000 species are recorded from India (Chandra \& Nema 2007). Extensive faunistic surveys, along with proper identification and documentation, at least to species and subspecies level, provide the most reliable data for conservation and management of different habitats.

Materials and Methods: Study area: The district of Tawang is located in Arunachal Pradesh, bordered by Tibet to the north, Bhutan to the southwest and the Sela ranges separate it from West Kameng District in the east. The district is situated at $27^{\circ} 33^{\prime} \mathrm{N}$ and $41^{\circ} 48^{\prime} \mathrm{E}$ between 2000-3000 m. A rapid faunistic survey or assessment was carried out in the study area which included the Lumla Forest Range including Bukhiyong, Thrillum, Thonglong, and a few localities of the Zemmethang Forest Ranges. The geographic coordinates of the collection localities are listed in Table 1.

Collection method: The sheet method was used, which allows collection of all the specimens individually without any damage. A white cloth sheet $\left(10^{\prime} \times 6^{\prime}\right)$ was hung between two vertical poles in such a way that it touched the surface and extended forward over the ground slightly away from direct source of light placed at such a point that the whole sheet from edge to edge brightly reflected the light. A 160 watt mercury vapour lamp was used as a light source through the night. Moths started collecting on

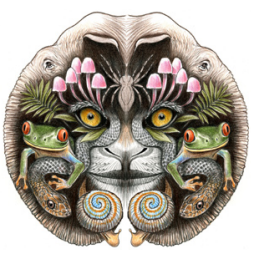

ISSN

Online 0974-7907 Print 0974-7893

\section{OPEN ACCESS} the sheet just after sunset between 1800-2300 hr, after that the abundance of moths slowly declined.

Identification: The moths collected from different localities were identified, and classified with the available literature (Hampson 1894-96; Bell \& Scott 1937) and their current nomenclature is based on LEPINDEX (Beccaloni et al. 2003). The hierarchy of different families of moths is based on the modern classification of insects above family level by Varshney (2003) and Van Nieukerken et al. (2011).

Results: The study revealed that a total of 102 species belonging to 81 genera, 24 subfamilies, 12 families under seven superfamilies (Table 2 ).

Discussion and Conclusions: The moth fauna of the family Arctiidae of Arunachal Pradesh was studied by Arora \& Chaudhury (1982). Kirti et al. (2005) inventoried 105 species of the family Arctiidae from northeastern India. But, the information on moth fauna of Tawang District has been very poorly studied and very few species are reported. In the present study, more than 250 morpho-species of moths were collected and 102 species, 81 genera under 12 diverse families were identified (Annexure 1). The family Geometridae dominated with $48 \%$ of the total species recorded, followed by the families Erebidae (26\%), Drepanidae (8\%), Crambidae (7\%), Uraniidae (3\%), Lasciocampidae

DOI: http://dx.doi.org/10.11609/JoTT.o2718.966 | ZooBank: urn:Isid:zoobank.org:pub:14139C6D-2D94-497D-9E44-8245FD5F468D

Editor: Peter Smetacek, Butterfly Research Centre, Bhimtal, India.

Date of publication: 26 January 2013 (online \& print)

Manuscript details: Ms \# 02718 | Received 24 February 2012 | Final received 10 October 2012 | Finally accepted 12 November 2012

Citation: Chandra, K. \& S. Sambath (2013). Moth diversity of Tawang District, Arunachal Pradesh, India. Journal of Threatened Taxa 5(1): 3565-3570. doi:10.11609/ JoTT.02718.966

Copyright: (c) Chandra \& Sambath 2013. Creative Commons Attribution 3.0 Unported License. JoTT allows unrestricted use of this article in any medium, reproduction and distribution by providing adequate credit to the authors and the source of publication.

Funding: None.

Competing Interest: None.

Acknowledgements: The authors are highly grateful to the Director, Zoological Survey of India, Kolkata for providing necessary field facilities. Sincere thanks are also due to D.F.O. Lumla and Zemmethang Range, State Forest Department, Tawang District, Arunachal Pradesh for providing helps during the course of study. Thanks are also due to Dr. G. Maheshwaran, Scientist C and Officer-in-Charge, Zoological Survey India, Arunachal Pradesh Field Station for constant cooperation during the period of field study. 
Table 1. Geographic coordinates of collection localities in Tawang District

\begin{tabular}{|l|c|c|c|}
\hline Collection localities & $\begin{array}{c}\text { Latitude } \\
\text { (N) }\end{array}$ & $\begin{array}{c}\text { Longitude } \\
\text { (S) }\end{array}$ & $\begin{array}{c}\text { Altitude } \\
(\mathrm{m})\end{array}$ \\
\hline $\begin{array}{l}\text { Lumla Circuit House (Camp-I) } \\
\text { (29.ix.2009-06.ix.2009) }\end{array}$ & $27^{\circ} 31^{\prime} 45^{\prime \prime}$ & $91^{\circ} 42^{\prime} 37^{\prime \prime}$ & 2408 \\
\hline Thonglong (03.x.2009) & $27^{0} 34^{\prime} 34^{\prime \prime}$ & $91^{\circ} 47^{\prime} 33^{\prime \prime}$ & 2357 \\
\hline Thrillum (03.x.2009) & $27^{\circ} 32^{\prime} 45^{\prime \prime}$ & $91^{\circ} 44^{\prime} 51^{\prime \prime}$ & 2355 \\
\hline $\begin{array}{l}\text { Zemmethang (tourist lodge) } \\
\text { (Camp-II) (07.x.2009-15.x.2009) }\end{array}$ & $27^{\circ} 42^{\prime} 38^{\prime \prime}$ & $91^{\circ} 43^{\prime} 27^{\prime \prime}$ & 2190 \\
\hline
\end{tabular}

(2\%), Sphingidae (2\%). The other moth families such as Pyralidae, Zygaenidae, Bombycidae, Saturniidae, and Notodontidae are represented each by $1 \%$. The family Geometridae is one of the largest families of the order Lepidoptera and is represented by Geometrinae, Ennominae, Larentiinae and Sterrhinae. The increase in distribution of the members of moths under Larentiinae is related to latitude (Ghosh 2003), and in the tropics similar trends are observed with increase in altitude (Holloway 1993, 1997). However, in the present study, the subfamily Ennominae outnumbers the members of other subfamilies and families. Overall higher species richness and diversity was recorded in the forest regions of Zemmethang more than the other localities of the study areas. The results from this study can be used to make decisions on the conservation of natural resources management especially for insect biodiversity. Hence, intensive surveys with long term monitoring programmes will help to identify the status of the species with the help of IUCN categories for the conservation and management of biodiversity.

\section{REFERENCES}

Alfred, J.R.B., A.K. Das \& A.K. Sanyal (1998). Faunal Diversity in India. ENVIS Centre Zoological Survey of India, Kolkata: 311-318.

Arora, G.S. \& M. Chaudhury (1982). On the Lepidopterous Fauna of Arunachal Pradesh \& Adjoining areas of Assam in North-East India (Family: Arctiidae), Zoological Survey of India, Tech. Mono. No. 6: 1-63pp.

Beccaloni, G.W., M.J., Scoble, G.S. Robinson, A.C. Downton \& S.M. Lucas (2003). Lepindex - The Global Lepidoptera Names Index: An online website published by the Natural History Museum, London.

Bell, T.R.D. \& F.B. Scott (1937). The Fauna of British India including Ceylon and Burma-Moths 5. Taylor and Francis, London, 537pp.

Chandra, K. \& D.K. Nema (2007). Fauna of Madhya Pradesh (including Chhattisgarh) part-I, State Fauna Series 15: 347. Published by Director, Zoological Survey of India, Kolkata.

Ghosh, S.K. (2003). Insecta: Lepidoptera: Heterocera: Geometridae, State Fauna Series 9, Fauna of Sikkim (Part-4): 217-342.

Hampson, G.F. (1892). The Fauna of British India including Ceylon and Burma-Moths 1. Taylor and Francis, London, 527pp.

Hampson, G.F. (1894). The Fauna of British India including Ceylon and Burma-Moths 2. Taylor and Francis, London, 528pp.
Table 2. The diversity of moths in Tawang District, Arunachal Pradesh, India as recorded in the present study

\begin{tabular}{|c|c|c|c|c|c|}
\hline & \multirow[b]{2}{*}{ Superfamily } & \multirow[b]{2}{*}{ Family } & \multicolumn{3}{|c|}{ Number of } \\
\hline & & & Subfamily & $\begin{array}{l}\text { Genus/ } \\
\text { Genera }\end{array}$ & Species \\
\hline 1. & Zygaenoidea & Zygaenidae & Chalcosiinae & 1 & 1 \\
\hline \multirow{2}{*}{2.} & \multirow{2}{*}{ Pyraloidea } & Pyralidae & Pyralinae & 1 & 1 \\
\hline & & Crambidae & Spilomelinae & 7 & 7 \\
\hline \multirow{4}{*}{3.} & \multirow{4}{*}{ Drepanoidea } & \multirow{4}{*}{ Drepanidae } & Cyclidiinae & 1 & 1 \\
\hline & & & Drepaninae & 4 & 4 \\
\hline & & & Thyatirinae & 3 & 3 \\
\hline & & & Oretinae & 1 & 1 \\
\hline 4. & Lasiocampoidea & Lasiocampidae & Lasiocampinae & 2 & 2 \\
\hline \multirow{4}{*}{5.} & \multirow{4}{*}{ Bombycoidea } & Bombycidae & - & 1 & 1 \\
\hline & & Saturniidae & Saturniinae & 1 & 1 \\
\hline & & \multirow{2}{*}{ Sphingidae } & Sphinginae & 1 & 1 \\
\hline & & & Macroglossinae & 1 & 1 \\
\hline \multirow{6}{*}{6.} & \multirow{6}{*}{ Geometroidea } & \multirow{2}{*}{ Uraniidae } & Microniinae & 1 & 1 \\
\hline & & & Epipleminae & 2 & 2 \\
\hline & & \multirow{4}{*}{ Geometridae } & Geometrinae & 8 & 9 \\
\hline & & & Ennominae & 21 & 32 \\
\hline & & & Larentiinae & 5 & 7 \\
\hline & & & Sterrhinae & 1 & 1 \\
\hline \multirow{8}{*}{7.} & \multirow{8}{*}{ Noctuoidea } & Notodontidae & - & 1 & 1 \\
\hline & & \multirow{7}{*}{ Erebidae } & Arctiinae & 6 & 8 \\
\hline & & & Lithosiinae & 4 & 6 \\
\hline & & & Lymantriinae & 4 & 6 \\
\hline & & & Amphipyrinae & 1 & 1 \\
\hline & & & Erebinae & 3 & 3 \\
\hline & & & Hadeninae & 1 & 1 \\
\hline & & & Pantheinae & 1 & 1 \\
\hline \multicolumn{4}{|r|}{ Total } & 81 & 102 \\
\hline
\end{tabular}

Hampson, G.F. (1895). The Fauna of British India including Ceylon and Burma-Moths 3. Taylor and Francis, London, 546pp.

Hampson, G.F. (1896). The Fauna of British India including Ceylon and Burma-Moths 4. Taylor and Francis, London, 594pp.

Holloway, J.D. (1993). Moths of Borneo: The family Geometridae, subfamily Ennominae. Malayan Nature Journal 47: 1-309.

Holloway, J.D. (1997). The Moths of Borneo: Family Geometridae, subfamilies Sterrhinae and Larentiinae. Malayan Nature Journal 51: 1-242.

Kirti J.S., J.S. Sodhi \& N.S. Gill (2005). Inventory of Arctiidae of NorthEastern India (Arctiidae: Lepidoptera). Journal of Entomological Research 29(3): 243-249.

Van Nieukerken, E.J. et al. (2011). Order Lepidoptera Linnaeus, 1758. In: Zhang, Z.-Q. (ed.) 2011: Animal biodiversity: an outline of higherlevel classification and survey of taxonomic richness. Zootaxa 3148 : 212-221.

Varshney, R.K. (2003). Modern classification of Insecta above family level. Bionotes 5(3\&4) \& 6(1,2\&3): 61-62 \& 85-87; 9-11; 43-44; 67-69. 


\section{Annexure 1. List of taxa}

Order: Lepidoptera

Suborder: Glossata Fabricius, 1775

Infraorder: Heteroneura

Clade: Apoditrysia Minet, 1983

Superfamily: Zygaenoidea Latreille, 1809

Family: Zygaenidae Latreille, 1809

Subfamily: Chalcosiinae

1. Herpa subhyalina Moore, 1879 (Image 1)

Clade: Obtectomera Minet, 1986

Superfamily: Pyraloidea Latreille, 1809

Family: Pyralidae Latreille, 1809

Subfamily: Pyralinae

2. Vitessa suradeva Moore, 1860 (Image 2)

Family: Crambidae Latreille, 1810

Subfamily: Spilomelinae

3. Botyodes principalis Leech, 1889 (Image 3)

4. Bradina diagonalis (Guenee, 1854) (Image 4)

5. Maruca testulalis (Geyer, 1832) (Image 5)

6. Palpita asiaticalis Inoue, 1994

7. Parotis vertumnalis (Guenee, 1854)

8. Spoladea recurvalis (Fabricius, 1775) (Image 6)

9. Syllepta verecunda Warren, 1896

Clade: Macroheterocera Chapman, 1893'

Superfamily: Drepanoidea Boisduval, 1828

Family: Drepanidae Boisduval, 1828

Subfamily: Cyclidiinae

10. Cyclidia rectificata Walker, 1862 (Image 7)

Subfamily: Drepaninae

11. Drepana pallida Moore, 1879

12. Macrauzata fenestraria (Moore, 1867) (Image 8)

13. Macrocilix mysticata (Walker, 1863) (Image 9)

14. Thymistada tripunctata Walker, 1865

Subfamily: Thyatirinae

15. Gaurena florens, Walker, 1865

16. Habrosyne derasa (Linnaeus, 1767)

Subfamily: Oretinae

17. Oreta sanguinea (Moore, 1879)

Superfamily: Lasiocampoidea Harris, 1841

Family: Lasiocampidae Harris, 1841

Subfamily: Lasiocampinae

18. Euthrix laeta (Walker, 1855)

19. Trabala vishnou Lefebvre, 1827 (Image 10)

Superfamily: Bombycoidea Latreille, 1802

Family: Bombycidae Latreille, 1802

20. Bombyx huttoni Westwood, 1847

Family: Saturniidae Boisduval, 1837

Subfamily: Saturniinae

21. Caligula simla Westwood, 1847

Family: Sphingidae Latreille, 1802

Subfamily: Sphinginae

Tribe: Acherontiini

22. Acherontia lachesis (Fabricius, 1798) (Image 11)

Subfamily: Macroglossinae

Tribe: Macroglossini

23. Cechenena lineosa (Walker, 1856)

Superfamily: Geometroidea Leach, 1815

Family: Uraniidae Leach, 1815

Subfamily: Microniinae

24. Acropteris iphiata Guen'ee, 1857 (Image 12)
Subfamily: Epipleminae

25. Dysaethria fulvilinea Hampson, 1895

26. Europlema himala (Butler, 1880) (Image 13)

Family: Geometridae Leach, 1815

Subfamily: Geometrinae

27. Agathia hilarata (Guenee, 1858)

28. Chlorissa gelida (Butler, 1889)

29. Chlorochaeta pictipennis (Butler, 1880)

30. Comostola subtiliaria Bremer, 1864

31. Gelasma thetydaria (Guenee, 1857)

32. Hemithea graminea Hampson 1891.

33. Mixochlora parsinus (Butler, 1879)

34. Tanaorhinus dimissa (Walker, 1861)

35. Tanaorhinus luteoviridata (Walker, 1861) (Image 14)

Subfamily: Ennominae

36. Abraxas (Abraxas) conferta Swinhoe, 1893

37. Abraxas (Calospilos) pusilla (Butler, 1880)

38. Abraxas (Calospilos) martaria (Guenee, 1857)

39. Abraxas (Calospilos) neomartaria Inoue, 1970

40. Alcis arisema Prout, 1934 (Image 15)

41. Aplochlora vivilaca (Walker, 1861)

42. Arichanna lapsariata (Walker, 1862)

43. Arichanna maculata (Moore, 1869)

44. Ascotis selenaria Schiffermuller, 1775

45. Campaea haliaria (Walker, 1861)

46. Chorodna vulpinaria Moore, 1867

47. Cleora acaciaria (Boisduval, 1833)

48. Dalima schistacearia Moore, 1867

49. Elphos hymenaria Guenee, 1857

50. Godonela emersaria Walker, 1861

51. Godonela nora (Walker, 1861)

52. Heterocallia temeraria Swinhoe, 1891

53. Hypochrosis quadraria Warren

54. Hypochrosis rufescens (Butler, 1880)

55. Loxaspilates sp. Warren, 1893

56. Medasina contaminata (Moore, 1887)

57. Medasina creataria (Guenee, 1857)

58. Nothomiza dentisignata (Moore, 1867)

59. Odontopera obliquaria (Moore, 1867)

60. Odontopera similaria (Moore, 1888)

61. Ourapteryx ebuleata Guenee, 1858

62. Ourapteryx picticaudata Walker, 1860

63. Ourapteryx primularis Butler, 1886

64. Ourapteryx sciticaudaria Walker, 1862

65. Opisthograptis molleri Warren, 1893

66. Plutodes costatus (Butler, 1886)

67. Sirinopteryx rufivinctata (Walker, 1862)

Subfamily: Larentiinae

68. Amnesicoma albiseriata (Warren, 1893)

69. Hydrelia ornata (Moore, 1867)

70. Pericallia viridescens (Warren, 1894)

71. Psyra angulifera Walker, 1867

72. Psyra spurcataria (Walker, 1862)

73. Xandrames albofasciata Moore, 1867 (Image 16)

74. Xandrames latiferaria Walker, 1860

Subfamily: Sterrhinae

75. Calothysanis responsaria Moore, 1888

Superfamily: Noctuoidea Latreille, 1809

Family: Notodontidae Stephens, 1829

76. Allata argentifera (Walker, 1862)

Family: Erebidae Leach, 1815

Subfamily: Arctiinae

77. Agylla ramelana (Moore, 1865) (Image 17)

78. Creatonotus transiens (Walker, 1855) (Image 18)

79. Mangina argus (Kollar, 1844) 
80. Nyctemera adversata (Schaller, 1788) (Image 19) 81. Nyctemera arctata (Walker, 1764) (Image 20)

82. Spilarctia obliqua Walker, 1855

83. Spilosoma comma (Walker, 1856)

84. Spilosoma dalbergiae (Moore, 1888) (Image 21)

Subfamily: Lithosiinae

85. Barsine cuneonotata (Walker, 1855)

86. Barsine inflexa (Moore, 1878)

87. Chrysorabdia bivitta (Walker, 1856) (Image 23)

88. Cyana divakara (Moore, 1865)

89. Cyana signa (Walker, 1854) (Image 22)

90. Eilema colon Moeschler, 1872

\section{Subfamily: Lymantriinae}

91. Arctornis divisa (Walker, 1855)

92. 'Euproctis' inconcisa (Walker, 1865)

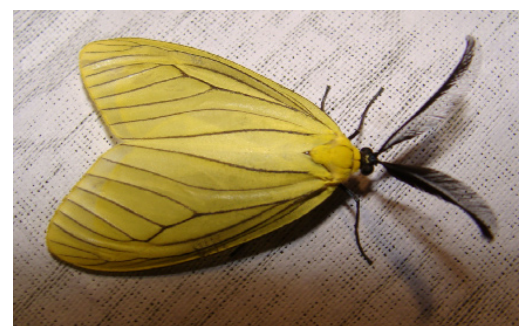

Image 1. Herpa subhyalina Moore (Zygaenidae)

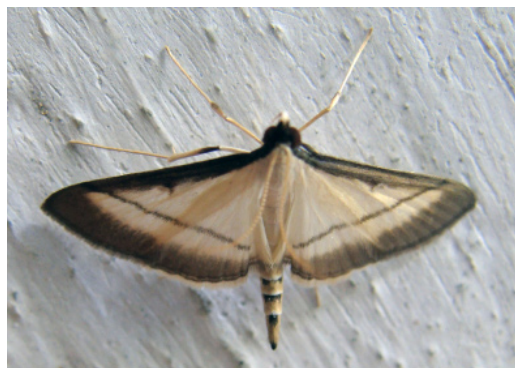

Image 4. Bradina diagonalis (Guenee) (Crambidae)

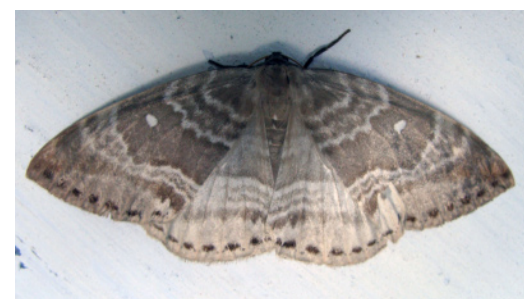

Image 7. Cyclidia rectificata Walker (Drepanidae)

(C) for all images Kailash Chandra \& S. Sambath
93. 'Euproctis' quadrangularis (Moore, 1879)

94. 'Euproctis' similis (Moore, 1879)

95. Lymantria ascetria Hubner, 1821

96. Numenes patrana Moore, 1859

Subfamily: Amphipyrinae

97. Amphipyra cupreipennis Moore, 1882

Subfamily: Erebinae

98. Arcte polygrapha Kollar, 1844 (Image 24)

99. Catocala patala Felder, 1874

100. Hypopyra unistrigata (Guenee, 1952)

Subfamily: Hadeninae

101. Actinotia intermediata (Bremer, 1861) (Image 25)

Subfamily: Pantheinae

102. Trichosea diffusa (Warren, 1913)

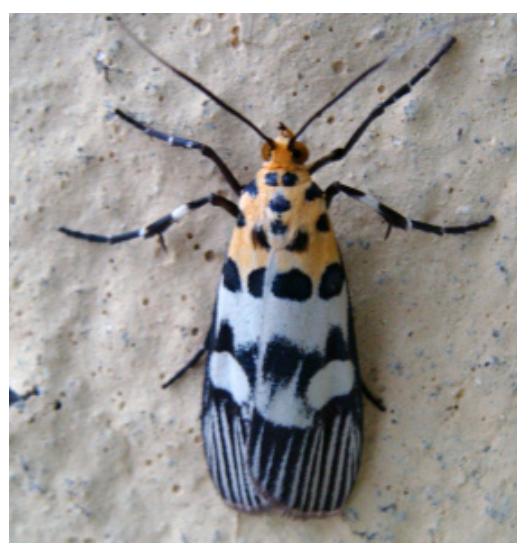

Image 2. Vitessa suradeva Moore (Pyralidae)

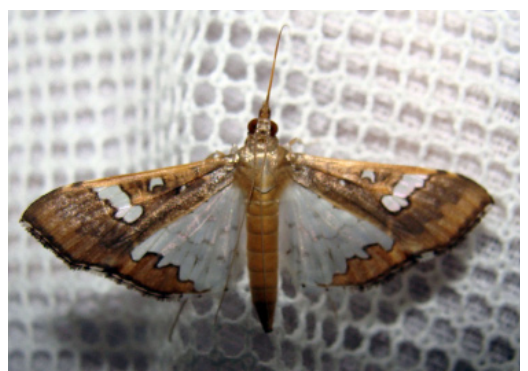

Image 5. Maruca testulalis (Geyer) (Crambidae)

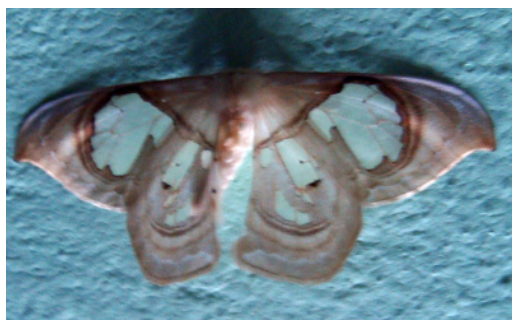

Image 8. Macrauzata fenestraria (Moore) (Drepanidae)

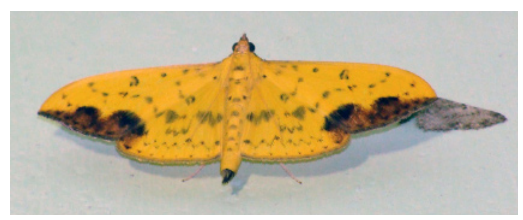

Image 3. Botyodes principalis Leech (Crambidae)

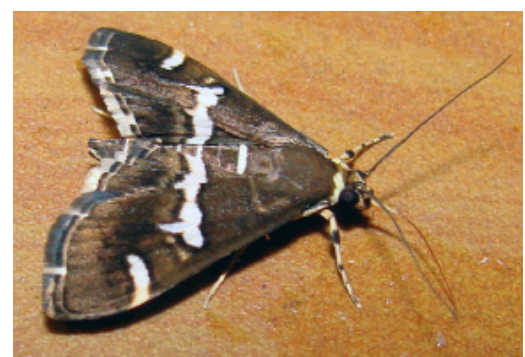

Image 6. Spoladea recurvalis (Fabricius) (Crambidae)

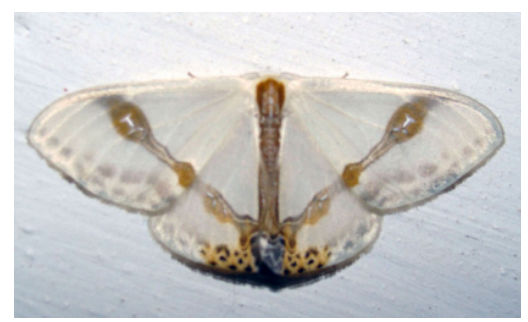

Image 9. Macrocilix mysticata (Walker) (Drepanidae) 


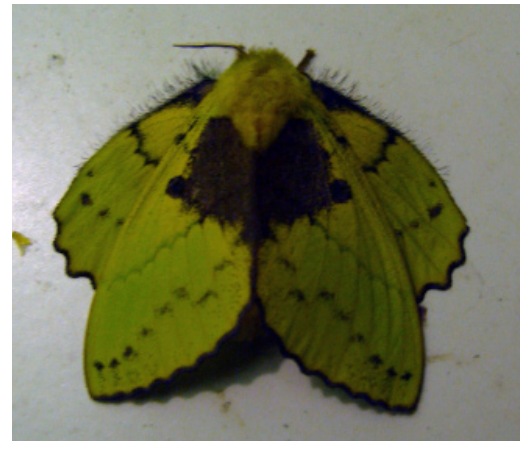

Image 10. Trabala vishnou Lefebvre (Lasiocampidae)

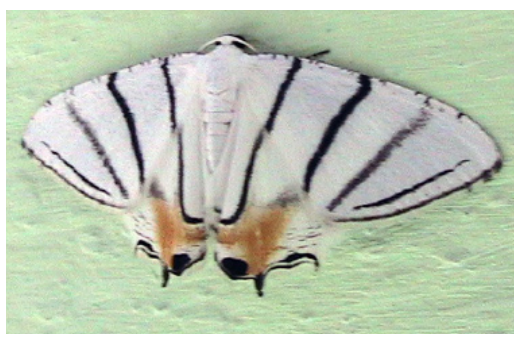

Image 13. Europlema himala (Butler) (Uraniidae)

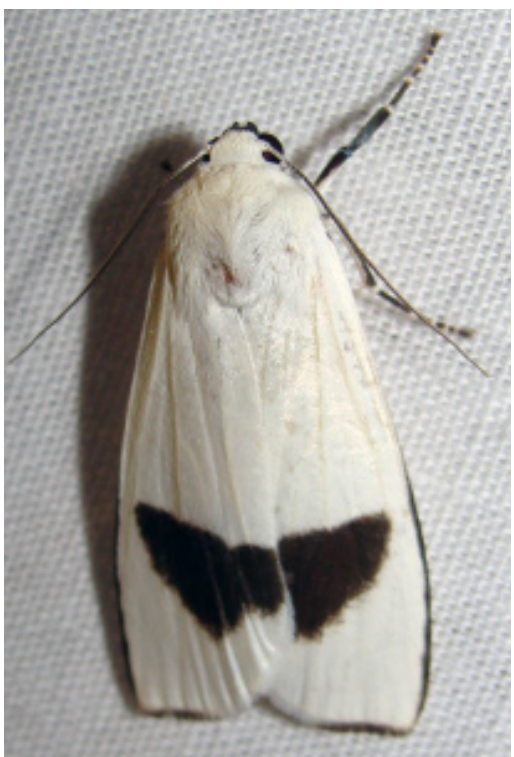

Image 17. Agylla remelana (Moore) (Erebidae)

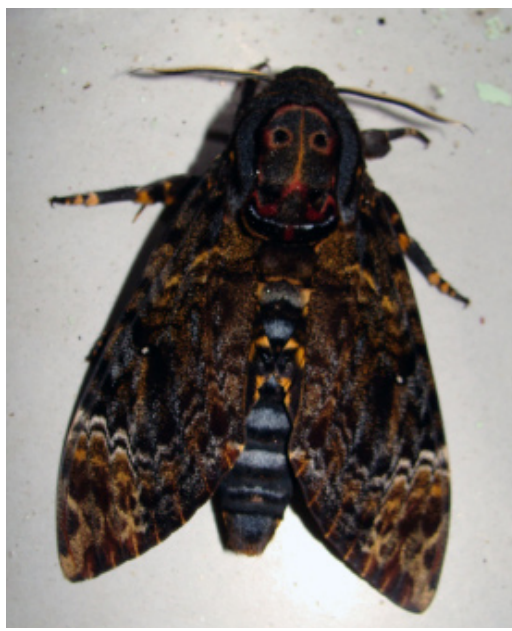

Image 11. Acherontia lachesis (Fabricius) (Sphingidae)

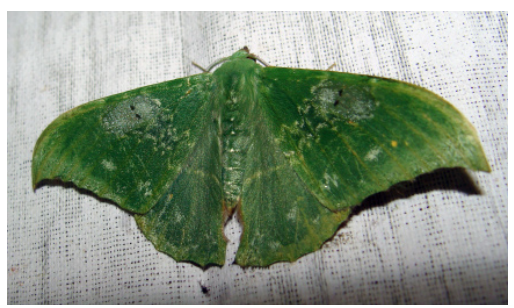

Image 14. Tanaorrhinus /uteoviridata (Walker) (Geometridae)

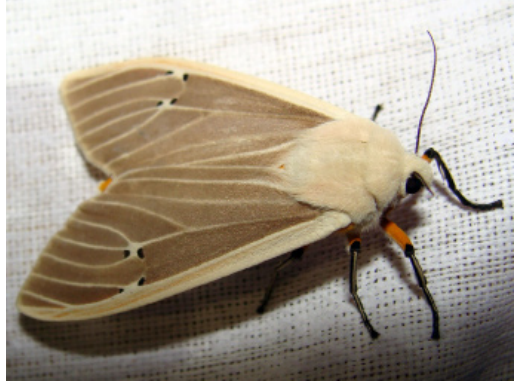

Image 18. Creatonotus transiens (Walker) (Erebidae) (Image 18)

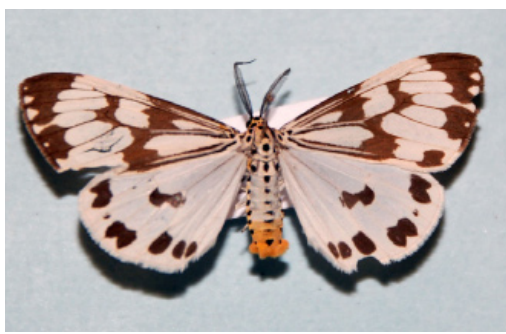

Image 19. Nyctemera adversata (Schaller) (Erebidae)

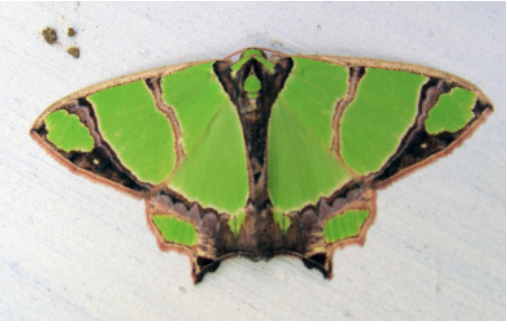

Image 12. Agathia hilarata (Guenee) (Geometridae)

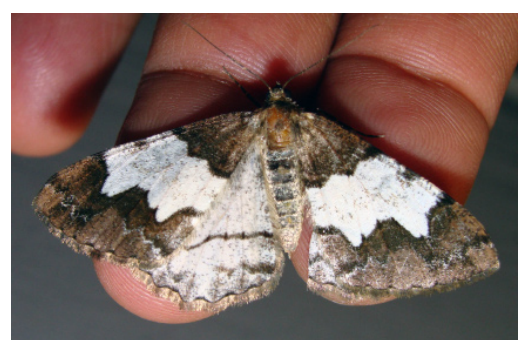

Image 15. Alcis arisema Prout (Geometridae)

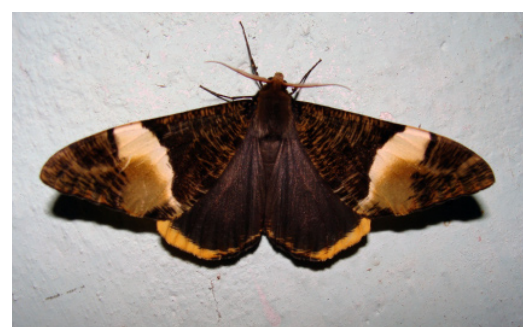

Image 16. Xandrames albofasciata Moore (Geometridae)

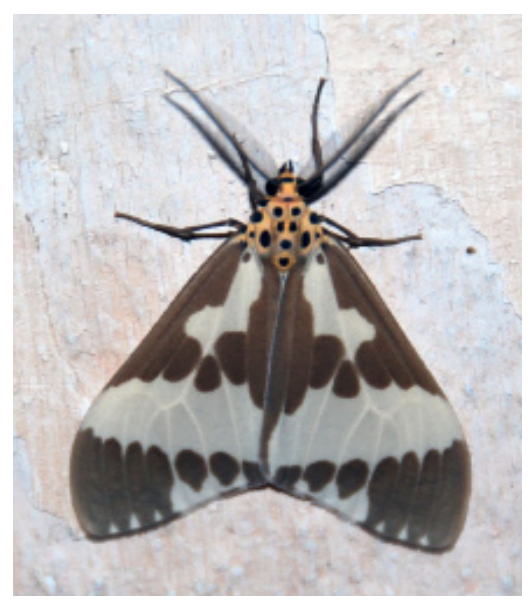

Image 20. Nyctemera arctata (Walker) (Erebidae) 


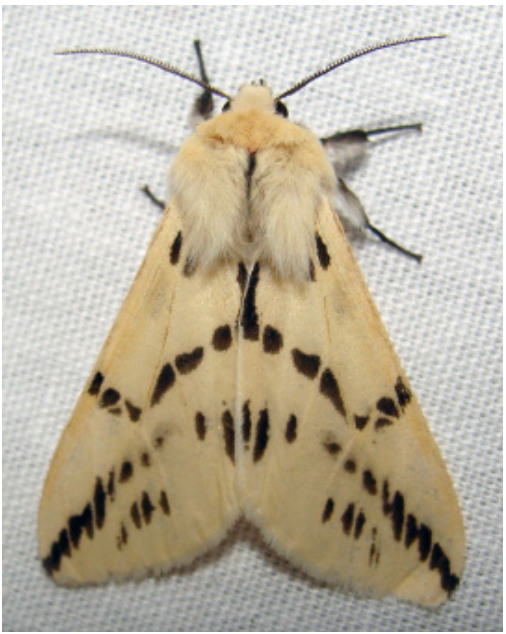

Image 21. Spilosoma dalbergiae (Moore) (Erebidae)

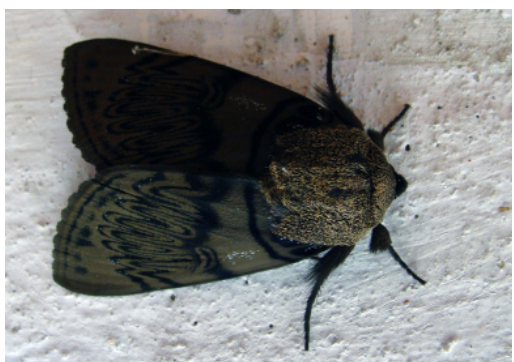

Image 24. Arcte polygrapha Kollar (Erebidae)
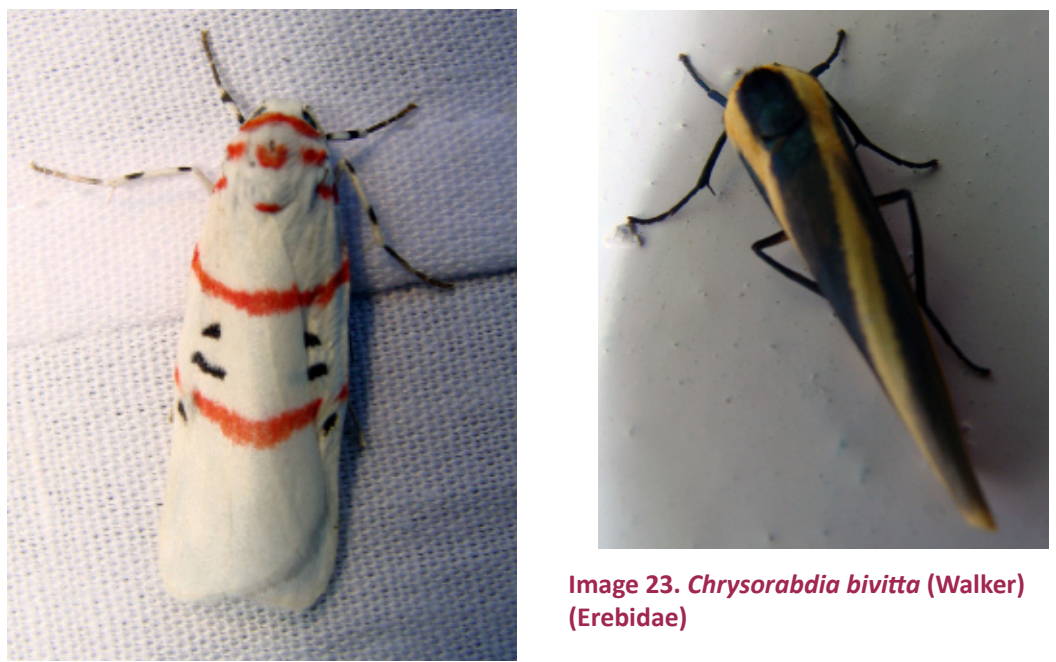

Image 23. Chrysorabdia bivitta (Walker) (Erebidae)

Image 22. Cyana signa (Walker) (Erebidae)

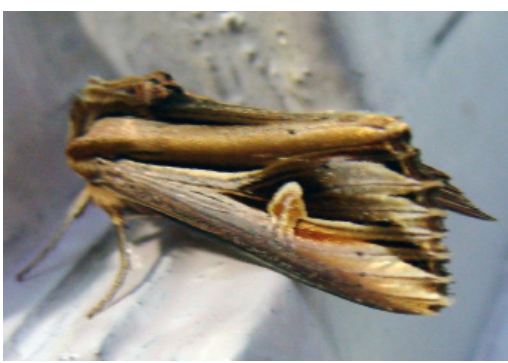

Image 25. Actinotia intermediata (Bremer) (Erebidae) 